\title{
COUETTE FLOW OF A GAS BY ELZAKI TRANSFORM
}

\author{
B. Vennila \\ Department of Mathematics \\ Faculty of Science \& Humanities \\ SRM University \\ Kattankulathur, 603 203, Tamil Nadu, INDIA
}

\begin{abstract}
Unsteady couette flow of a gas between two parallel infinite plates have been studied in this paper. We proposed a new transform technique called Elzaki transform. Analytical solution is obtained for the equation of motion by this method. Although the Elzaki transform have a close connection with the Laplace transform, Elzaki transform is easy to employ and has got several advantages over Laplace transform method.
\end{abstract}

AMS Subject Classification: 76U05, 76U99

Key Words: Elzaki transform method, differential equation, viscosity coefficient, Couette flow

\section{Introduction}

Couette flow is frequently used in Physics and Engineering. Couette flow between parallel plates is a classical problem that has an important applications in power generators and pumps etc. Several investigators have been done this type of flow. But still it seems that not much work have been done. The Taylor Couette flow using DNS/SUV method was studied by Tuliszka-Szhilko et al [1]. Haider Zaman et al [2] discussed the Couette flow problem for an unsteady MHD fourth - grade fluid with Hall currents. Couette flow of a binary mixture of rigid sphere gases described by the linearized Boltzman equation was discussed by Gracia et al [3]. Risso et al[4] analysed the dilute gas Cou-

Received: $\quad$ February 19, 2017

Revised: $\quad$ August 30, 2017

Published: September 14, 2017 (c) 2017 Academic Publications, Ltd. url: www.acadpubl.eu 
ette flow theory and molecular dynamics simulation. Debashish Sircar et al [5] discussed the flow and heat transfer in a micro-channel gas-liquid couette flow. The unsteady and steady velocity profile for couette flow was presented and solved exactly using the numerical method by Rihan [6]. Tarig Elzaki et al [7] studied the fundamental properties and application of Elzaki transform. The connection between Laplace and Elzaki transform was discussed by Tarig Elzaki [8]. Hayat et al [9] used Laplace transform method to determine the analytical solutions of couette flows of a second grade fluid. Erdogan et al [10] solved unsteady couette flow for viscous fluid by Laplace transform method. Vennila [11] studied Elzaki Transform for Rotating Fluid.

\section{Mathematical Analysis}

Consider the motion of a fluid between two infinite parallel plates. At time $t<0$, both the fluid and the plates are assumed to be at rest. At $t=0$, the lower plate begins to move in its own plane with a velocity proportional to $\mathrm{U}$ and the upper plate at a distance $l$ apart, remains fixed.

We choose a Cartesian coordinate system of axes with $\mathrm{x}$-axis along the lower plate, $\mathrm{y}$-axis perpendicular to it at $\mathrm{z}$-axis lying on the plate.

The equation of motion can be written as

$$
\begin{aligned}
\frac{\partial u}{\partial t}+(u . \nabla) u & =-\frac{1}{\rho} \operatorname{grad} p+\nu \nabla^{2} u \\
\operatorname{div}(u) & =0
\end{aligned}
$$

Equation (1) reduced to

$$
\frac{\partial u}{\partial t}=\nu \frac{\partial^{2} u}{\partial y^{2}}
$$

Boundary conditions are

$$
\left\{\begin{array}{l}
u=U, y=0 \text { for } t>0 \\
u=0 \text { at } y=l \text { for } t>0 \\
u=0 \text { in } 0 \leq y \leq l \text { for } t \leq 0
\end{array}\right.
$$

Let $\bar{T}(y, v)$ be the Elzaki transform of $u(y, t)$, (i.e.) $E[u(y, t)]=\bar{T}(y, v)$.

Then taking the Elzaki transform on both side of equation (2), we have

$$
\frac{d^{2} \bar{T}}{d y^{2}}-\frac{1}{\nu}\left\{\frac{\bar{T}(y, v)}{v}-v u(y, 0)\right\}=0
$$


Using condition equation (3) in equation (4), we obtain

$$
\frac{d^{2} \bar{T}}{d y^{2}}-\frac{1}{\nu v} \bar{T}(y, v)=0
$$

On solving the above equation, we get

$$
\bar{T}(y, v)=C_{1} e^{\left(\frac{1}{\sqrt{\nu v}}\right) y}+C_{2} e^{-\left(\frac{1}{\sqrt{\nu v}}\right) y}
$$

Applying Elzaki transform of the boundary conditions, we obtain

$$
\bar{T}(0, v)=U v^{2}, \bar{T}(l, v)=0, \bar{T}(y, 0)=0
$$

Apply equation (7) in equation (6), we get

$$
C_{1}=\frac{U v^{2}}{1-e^{\frac{2 l}{\sqrt{\nu v}}}} ; C_{2}=-\frac{U v^{2} e^{\frac{l}{\sqrt{\nu v}}}}{1-e^{\frac{2 l}{\sqrt{\nu v}}}}
$$

Substituting these values in equation $((6))$, we obtain

$$
\begin{gathered}
\bar{T}(y, v)=-U v^{2}\left[\left.\frac{e^{\frac{-l}{\sqrt{\nu v}}} e^{\frac{l}{\sqrt{\nu v}}} y}{e^{\frac{l}{\sqrt{\nu v}}}-e^{\frac{-l}{\sqrt{\nu v}}}}\right|^{2}+U v^{2}\left[\frac{e^{\frac{l}{\sqrt{\nu v}}} e^{-\left(\frac{l}{\sqrt{\nu v}}\right) y}}{e^{\frac{l}{\sqrt{\nu v}}}-e^{\frac{-l}{\sqrt{\nu v}}}}\right]\right. \\
\bar{T}(y, v)=U v^{2}\left[\frac{\sinh \left(\frac{l-y}{\sqrt{\nu v}}\right)}{\sinh \left(\frac{l}{\sqrt{\nu v}}\right)} \mid\right. \\
u(y, t)=E^{-1}\left\{U v^{2}\left[\frac{\sinh \left(\frac{l-y}{\sqrt{\nu v}}\right)}{\sinh \left(\frac{l}{\sqrt{\nu v}}\right)} \mid\right\}\right.
\end{gathered}
$$

The inverse transform $E^{-1}$ of the right hand side terms in equation (8) may be determined by recalling a standard result as $[7,8]$

$$
E^{-1}(\bar{T}(y, v))=\sum \text { residue of }\left[e^{s T} s \bar{T}\left(y, \frac{1}{s}\right)\right]
$$

(i.e.) $\bar{T}\left(y, \frac{1}{s}\right)$ has pole at $s=0$ and other pole at $s=\frac{-n^{2} \pi^{2} \nu}{l^{2}}, n=$ $0, \pm 1, \pm 2, \cdots$. 
Now we calculate residues one by one.

Residue of $e^{s T} s \bar{T}\left(y, \frac{1}{s}\right)$ at $s=0$ is

$$
\lim _{s \rightarrow 0} \frac{d}{d s}\left\{\frac{(s-0)^{2} e^{s t} s \sinh \left(\sqrt{\frac{s}{\nu}}(l-y)\right)}{s^{2} \sinh \left(\sqrt{\frac{s}{\nu}}\right) l}\right\}=\frac{l-y}{l} .
$$

Residue of $e^{s T} s \bar{T}\left(y, \frac{1}{s}\right)$ at $s=s_{n}$ is

$$
\begin{aligned}
\lim _{s \rightarrow s_{n}} \frac{s e^{s t} \sinh \left(\sqrt{\frac{s}{\nu}}(l-y)\right)}{s^{2} \frac{d}{d s}\left[\sinh \left(\sqrt{\frac{s}{\nu}}\right) l\right]} \\
=\frac{e^{-\left(\frac{n^{2} \pi^{2} \nu}{l^{2}}\right) t} \sinh \left(\frac{n \pi i}{l}(l-y)\right) 2 \sqrt{\nu}}{\frac{\ln \pi i \sqrt{\nu}}{l} \cosh \left(\frac{n \pi i}{l} \frac{\sqrt{\nu}}{\sqrt{\nu}} l\right)}=-\frac{2 e^{-\left(\frac{n^{2} \pi^{2} \nu}{l^{2}}\right) T} \sin \frac{n \pi y}{l}}{n \pi} .
\end{aligned}
$$

Putting all these residues in (11), we receive

$$
u(y, t)=U\left\{\frac{l-y}{l}+\frac{\sum_{n=1}^{\infty}(-2) e^{-\left(\frac{n^{2} \pi^{2} \nu}{l^{2}}\right) t} \sin \frac{n \pi y}{l}}{n \pi}\right\} .
$$

\section{Conclusion}

In this paper analytical solution was obtained for the equation of motion for unsteady couette flow between two parallel infinite plates one of them was stationary and the other plate moved with constant velocity by Elzaki transform method.

\section{References}

[1] E. Tuliszka-Sznitko, L. Kietczeuski, Numerical Investigations of Taylor-Couette Flow using DNS/SUV Method, Computational Methods in Science and Technology, 21, No. 1 (2015), 211-219, doi: 10.1291/cmst.2015.21.04.005. 
[2] Haider Zaman, Tarique Akhas, Arif Sohail, Azhar Ali, Couette flow problem for an Unsteady MHD Fourth Grade Fluid with Hall Currents, Journal of Applied Mathematics and Physics, 2, No. 4 (2014), 1-10, doi: 10.4236/Jamp.2014-24001.

[3] R.P.M. Garcia, C.E. Siewert, Couette flow of a binary mixture of Rigid-Sphere Gases described by the Linearized Boltzmann Equation, European Journal of MechanicsB/Fluids, 27 (2008), 823-836, doi: 10.1016/j.euromechflu.2008.01.004.

[4] D. Risso, P. Cardero, Dilute Gas Couette Flow Theory and Molecular Dynamics Simulation, Physical Review, 56 (1997), 489-496, doi: 10.1103/PhysRevE.56.489.

[5] Debashish Sircar, Flow and Heat Transfer in a Micro-channel Gas-Liquid Couette Flow, International Journal of Heat and Mass transfer, 54, 2913-2920, doi: 10.1016/j.ijheatmasstransfer.2011.03.007.

[6] Pallavi P. Chopade, S. Bhuma Devi, Applications of Elzaki transform to ordinary Partial Differential Equations, International Journal of Advanced Research in Computer Science and Software Engineering, 5, 3(2015), 38-41.

[7] Tarig M. Elzaki, Salih M. Elzaki, On the connections between Laplace and Elzaki transforms, Advances in Theoretical and Applied Mathematics, 6, No. 1 (2011), 1-10.

[8] Tarig M. Elzaki, Salih M. Elzaki, Elsayed A. Elnour, On the Integral Transform Elzaki Transform Fundamental Properties Investigations and Applications, Global Journal of Mathematical Science, 4, 1 (2012), 15-23.

[9] T. Hayat, M. Masood Khan, Ayub, A.M. Siddiqui, The Unsteady Couette Flow of a Second grade Fluid in a Layer of Porous Medium, Arch. Mech., 57, No. 5 (2005), 405416.

[10] M.E. Erdogan, On the Unidirectional Flows Generated by Impulsive Notion of a Boundary or Sudden Application of a Pressure Gradient, International Journal of Non-linear Mechanics, 37 (2002), 1091-1106, doi: org/10.1016/s0020-7462(01)00035-x.

[11] B. Vennila, Elzaki Transform for Rotating Fluid, International Journal of Pure and Applied Mathematics, 110, No. 3 (2016), 407-412, doi: 10.12732/ijpam.v110i3.2. 
